\title{
TRB3 reverses chemotherapy resistance and mediates crosstalk between endoplasmic reticulum stress and AKT signaling pathways in MHCC97H human hepatocellular carcinoma cells
}

\author{
YANG LI ${ }^{1,2}$, DANXI ZHU ${ }^{3}$, LIDAN HOU ${ }^{3}$, BIN HU $^{2}$, MIN XU $^{1,2^{*}}$ and XIANGJUN MENG ${ }^{3 *}$ \\ ${ }^{1}$ Department of Gastroenterology, Shanghai General Hospital of Nanjing Medical University; ${ }^{2}$ Department of Gastroenterology, \\ Shanghai General Hospital of Shanghai Jiaotong University, Shanghai 200080; ${ }^{3}$ Department of Gastroenterology, \\ School of Medicine, Shanghai Ninth People's Hospital of Shanghai Jiaotong University, Shanghai 200011, P.R. China
}

Received June 11, 2016; Accepted September 22, 2017

DOI: $10.3892 / \mathrm{ol} .2017 .7361$

\begin{abstract}
Tribbles homolog 3 (TRB3), a type of pseudokinase that contains a consensus serine/threonine kinase catalytic core structure, is upregulated in hepatocellular carcinoma. However, the effect of TRB3 expression in hepatocellular carcinoma and the molecular mechanisms underlying TRB3-mediated effects on tumorigenesis in hepatocellular carcinoma have not been fully elucidated. The present study focused on the effect of TRB3 expression in MHCC97H hepatocellular carcinoma cells and investigated the underlying molecular mechanisms in MHCC97H cells. In the present study, it was revealed that TRB3 was significantly overexpressed in the MHCC $97 \mathrm{H}$ hepatocellular carcinoma cell compared with L-02 normal hepatic cells. Under endoplasmic reticulum (ER) stress induced by thapsigargin and tunicamycin, the levels of TRB3, CCAAT/enhancer binding protein homologous
\end{abstract}

Correspondence to: Professor Min Xu, Department of Gastroenterology, Shanghai General Hospital of Shanghai Jiaotong University, 100 Haining Road, Hongkou, Shanghai 200080, P.R. China

E-mail: xuminmd@163.com

Professor Xiangjun Meng, Department of Gastroenterology, School of Medicine, Shanghai Ninth People's Hospital of Shanghai Jiaotong University, 639 Zhizaoju Road, Huangpu, Shanghai 200011, P.R. China

E-mail: xiangjunmeng@aliyun.com

*Contributed equally

Abbreviations: ER, endoplasmic reticulum; HCC, hepatocellular carcinoma; shRNA, short hairpin RNA; UPR, unfolded protein response; AKT, protein kinase B; pAKT, phosphorylated AKT; TG, thapsigargin; TU, tunicamycin; DMSO, dimethyl sulfoxide; CHOP, CCAAT/enhancer binding protein homologous protein; ATF4, activating transcription factor 4

Key words: endoplasmic reticulum stress, protein kinase B signaling pathway, tribbles homolog 3, CCAAT/enhancer binding protein homologous protein, hepatocellular carcinoma protein (CHOP), protein kinase B (AKT) and phosphorylated (p)AKT expression were upregulated. Furthermore, when the expression of TRB3 was silenced by short hairpin (sh)RNA, the survival of MHCC $97 \mathrm{H}$ hepatocellular carcinoma cells was increased. Notably, following transduction with lentiviral containing TRB3-shRNA, cell survival also increased after treatment with chemotherapy drug cisplatin. The present study demonstrated that knockdown of CHOP by shRNA was able to reduce TRB3 expression, and the knockdown of TRB3 markedly increased the level of pAKT. TRB3 was overexpressed in MHCC97H hepatocellular carcinoma cells, particularly under endoplasmic reticulum stress. Knockdown of TRB3 was able to increase cell survival. Therefore, TRB3 expression may induce apoptosis and reverse resistance to chemotherapy in MHCC $97 \mathrm{H}$ hepatic carcinoma cells. The present study suggests that TRB3 is a key molecule that mediates the crosstalk between ER stress and AKT signal pathways. Furthermore, the present study may provide further insight into the cancer biology of hepatocellular carcinoma and the development of anticancer drugs targeting the ER stress and AKT signaling pathways.

\section{Introduction}

Hepatocellular carcinoma (HCC) is one of the most common types of tumor worldwide, particularly in less developed countries $(1,2)$. During 2012, 745,500 hepatocellular carcinoma-associated mortalities worldwide were reported, and the mortality rate was predicted to increase in developed areas, including the USA and Europe $(1,2)$. It has been demonstrated that chemotherapy resistance is one of the most important reasons leading to HCC patient mortality (3). Therefore, investigating a novel biomarker for HCC treatment is necessary.

Tribbles homolog 3 (TRB3) is a type of pseudokinase, which contains a consensus serine/threonine kinase catalytic core structure but does not exhibit kinase activity (4). Previous studies verified that TRB3 was upregulated in hepatic carcinoma cells and was able to induce apoptosis in hepatic carcinoma cell as a downstream target of activating transcription factor 4 (ATF4)-CCAAT/enhancer binding protein homologous protein (CHOP), a well-known signaling pathway, 
which serves a key role in endoplasmic reticulum (ER) stress $(5,6)$. Conversely, TRB3 inhibits activation of protein kinase $\mathrm{B}(\mathrm{AKT})$ by binding with its threonine or serine activated site, which results in the activation of the downstream signal to induce cell apoptosis $(7,8)$. Furthermore, much of the research in the previous two decades has indicated that the AKT signaling pathway is closely associated with the occurrence and development of hepatic carcinoma $(9,10)$.

On this basis, in the present study, it was hypothesized that TRB3 possessed a novel function to connect ER stress with the AKT signaling pathway and induce apoptosis and chemotherapy resistance in $\mathrm{HCC}$ cells. In the present study, the expression levels of TRB3, CHOP, AKT and phosphorylated (p)AKT were primarily detected under normal conditions and ER stress respectively. Subsequently, cell apoptosis and resistance to chemotherapy induced by TRB3 knockdown were observed. Furthermore, the associations between TRB3, the marker proteins of ER stress, CHOP and AKT, in $\mathrm{MHCC} 97 \mathrm{H}$ cells were investigated.

\section{Materials and methods}

Cell lines. MHCC97H and L-02 cell lines were provided by the Central Laboratory of Shanghai First People's Hospital (Shanghai, China). The cells were cultured in Dulbecco's modified Eagle's medium (DMEM; Hyclone, GE Healthcare Life Sciences, Logan, UT, USA) supplemented with $10 \%$ fetal bovine serum (FBS; Gibco; Thermo Fisher Scientific, Inc., Waltham, MA, USA) in $5 \% \mathrm{CO}_{2}$ at $37^{\circ} \mathrm{C}$. MHCC $97 \mathrm{H}$ cells were treated with two drugs, thapsigargin (TG; $5 \mu \mathrm{mol} / \mathrm{l}$; Beijing Solarbio Science \& Technology Co., Ltd., Beijing, China) and tunicamycin (TU; $5 \mu \mathrm{g} / \mathrm{ml}$; Beijing Solarbio Science \& Technology Co., Ltd.) for $24 \mathrm{~h}$, and DMSO $(5 \mu \mathrm{g} / \mathrm{ml}$; Beijing Solarbio Science \& Technology Co., Ltd.) was used as the control.

Lentiviral transduction of short hairpin (sh)RNA and transduction efficient examination. Transduction was performed using a GenePharma Lentivirus Transduction kit (Shanghai GenePharma Co., Ltd., Shanghai, China) according to the manufacturer's protocol. MHCC $97 \mathrm{H}$ cells were plated in six-well plates at a density of $2 \times 10^{5}$ cells/well. Following $24 \mathrm{~h}$ at $37^{\circ} \mathrm{C}$, lentiviruses carrying four different TRB3/CHOP targeting shRNAs (TRB3-shRNA 778, shRNA 1057, shRNA1185, shRNA1608; CHOP-shRNA 342, shRNA 807, shRNA 814, shRNA 929) and negative control (NC) shRNA (Shanghai GenePharma Co., Ltd.) were added to the cells. The shRNA sequences are shown in Table I. The concentration of shRNA added was $1 \times 10^{8} \mathrm{U} / \mathrm{ml}(40 \mu \mathrm{l})$. The viral titer was $1 \mathrm{ml} /$ well. At the same time, polybrene was added to each well at a final concentration of $6 \mu \mathrm{g} / \mathrm{ml}$. Following incubation at $37^{\circ} \mathrm{C}$ for $12 \mathrm{~h}$, the viral supernatant was replaced with DMEM supplemented with $10 \%$ FBS. The infected cells were selected using $2 \mathrm{mg} / \mathrm{ml}$ puromycin (Santa Cruz Biotechnology, Inc., Dallas, TX, USA) following incubation at $37^{\circ} \mathrm{C}$ for $48 \mathrm{~h}$. Successful infection was confirmed by the expression of green fluorescent protein using an inverted fluorescence microscope (Leica DMI4000 B; Leica Microsystems GmbH, Wetzlar, Germany). After 72 and $96 \mathrm{~h}$ at $37^{\circ} \mathrm{C}$ the total RNA and total proteins were isolated using TRIzol ${ }^{\circledR}$ (Invitrogen; Thermo Fisher Scientific, Inc.) and SDS sample loading buffer (Sigma-Aldrich; Merck KGaA, Darmstadt, Germany) respectively to determine the expression levels of target genes and proteins by reverse transcription-quantitative polymerase chain reaction (RT-qPCR) and western blot analysis. The cells were infected every $12 \mathrm{~h}$ for 2 days.

Cell Counting kit-8(CCK-8) assay. A total of $1 \times 10^{4} \mathrm{MHCC} 97 \mathrm{H}$ cells in $100 \mu \mathrm{l}$ DMEM containing $10 \%$ FBS were seeded in a 96-well plate format in $5 \% \mathrm{CO}_{2}$ at $37^{\circ} \mathrm{C}$. A total of $1 \mathrm{ml} \mathrm{CCK}-8$ (Dojindo Molecular Technologies, Inc., Kumamoto, Japan) was mixed with $10 \mathrm{ml}$ DMEM supplemented with $10 \%$ FBS. Following transfection with TRB3-shRNA 1608 at $37^{\circ} \mathrm{C}$ for $48 \mathrm{~h}$ or treatment with cisplatin $(4 \mu \mathrm{g} / \mathrm{ml}$; Sangon Biotech Co., Ltd., Shanghai, China) at $37^{\circ} \mathrm{C}$ for $24 \mathrm{~h}$, the $\mathrm{MHCC} 97 \mathrm{H}$ cells were then transferred into DMEM containing $10 \%$ FBS at $37^{\circ} \mathrm{C}$ for 48,72 and $96 \mathrm{~h}$, the $100 \mu \mathrm{l}$ diluted CCK- 8 was added to each well and then absorbance at $450 \mathrm{~nm}$ following $1 \mathrm{~h}$ of cultivation at $37^{\circ} \mathrm{C}$ condition was determined. The absorbance at the $48 \mathrm{~h}$ was used as the standard.

$R T-q P C R$. The total mRNA from L-02 and MHCC97H cells was extracted using TRIzol ${ }^{\circledR}$ (Invitrogen; Thermo Fisher Scientific, Inc.) and reverse transcribed into cDNA using the Reverse Transcription kit from Takara Biotechnology Co., Ltd. (Dalian, China) according to the manufacturer's protocol. The process of reverse transcription was as follows: $37^{\circ} \mathrm{C}$ for $15 \mathrm{~min}, 85^{\circ} \mathrm{C}$ for $5 \mathrm{sec}$, then held at $4^{\circ} \mathrm{C}$ hold. The forward and reverse primers used are presented in Table II. PCR reactions were performed using a FTC3000 Real-Time PCR Detection System (Funglyn Biotech, Inc., Richmond Hill, ON, Canada) with SYBR Premix Ex Taq ${ }^{\mathrm{TM}}$ II (Takara Biotechnology Co., Ltd.). The conditions as follows: Pre-denaturation for $3 \mathrm{~min}$ at $95^{\circ} \mathrm{C}$, followed by 40 cycles of denaturation for $30 \mathrm{sec}$ at $95^{\circ} \mathrm{C}$, annealing for $30 \mathrm{sec}$ at $52^{\circ} \mathrm{C}$, extension for $30 \mathrm{sec}$ at $72^{\circ} \mathrm{C}$ and a final cycle extension at $72^{\circ} \mathrm{C}$ for $10 \mathrm{~min}$. The level of relative gene expression was evaluated using the $\Delta \Delta \mathrm{Cq}$ method (11), and expression was normalized to GAPDH. All samples were analyzed three times.

Western blot analysis. The cells were lysed using SDS sample loading buffer (Sigma-Aldrich; Merck KGaA, Darmstadt, Germany) for $30 \mathrm{~min}$ on ice. The protein concentration was determined using a BCA protein assay kit (Beyotime Institute of Biotechnology, Haimen, China). Equal amounts of protein extracts $(30 \mu \mathrm{g})$ were separated electrophoretically on 10 or $15 \%$ SDS-PAGE gels and transferred to a polyvinylidene fluoride membrane. The membrane was blocked using $5 \% \mathrm{w} / \mathrm{v}$ bovine serum albumin (Beyotime Institute of Biotechnology), dissolved in TBS with Tween-20 (TBST; $0.1 \%$ Tween-20) at room temperature for $1 \mathrm{~h}$ and incubated sequentially with primary antibodies for $8 \mathrm{~h}$ at $4^{\circ} \mathrm{C}$ and peroxidase-conjugated secondary antibodies for $1 \mathrm{~h}$ at $25^{\circ} \mathrm{C}$. The antibodies used were as follows: Rabbit TRB3 polyclonal antibody (1:2,000; cat no. A2346; ABclonal Biotech Co., Ltd., Woburn, MA, USA), rabbit CHOP polyclonal antibody (1:5,000; cat no. A0221; ABclonal Biotech Co., Ltd.), rabbit AKT polyclonal antibody (1:1,000; cat no. A0001; ABclonal Biotech Co., Ltd.), rabbit pAKT-S473 polyclonal antibody (1:1,000; cat no. AP0098; ABclonal Biotech Co., Ltd.), rabbit GAPDH antibody (1:10,000; cat no. AB21612; ABclonal 
Table I. Primer sequences for genes used in reverse transcription-quantitative polymerase chain reaction.

\begin{tabular}{ll}
\hline Primer & \multicolumn{1}{c}{ Sequence (5'-3') } \\
\hline GAPDH & \\
Forward & CATGAGAAGTATGACAACAGCCT \\
Reverse & AGTCCTTCCACGATACCAAAGT \\
TRB3 & \\
Forward & GCTGCCAACAGTGGATTGA \\
Reverse & GCTTCTGCCTTTCTCCCTTCT \\
AKT & \\
Forward & GGCACATTAAGATCACAGACTTCG \\
Reverse & TCATTGTCCTCCAGCACCTC \\
CHOP & \\
Forward & TGAACGGCTCAAGCAGGAA \\
Reverse & AGGGTCACATCATTGGCACTA \\
\hline
\end{tabular}

AKT, protein kinase $\mathrm{B}$; TRB3, tribbles homolog 3; CHOP, CCAAT/enhancer binding protein homologous protein.

Table II. Sequences of shRNAs.

\begin{tabular}{lc}
\hline shRNA & \multicolumn{1}{c}{ Sequence (5'-3') } \\
\hline TRB3 & \\
shRNA 778 & GCACTGAGTATACCTGCAAGG \\
shRNA 1057 & AGCTGTGTCGCTTTGTCTTCG \\
shRNA1185 & GGACCTGAGATACTCAGCTCA \\
shRNA1608 & GCCAACAGTGGATTGAGTTTG \\
CHOP- & \\
shRNA 342 & CAGCTGAGTCATTGCCTTTCT \\
shRNA 807 & GAGCTCTGATTGACCGAATGG \\
shRNA 814 & GATTGACCGAATGGTGAATCT \\
shRNA 929 & GCCAATGATGTGACCCTCAAT
\end{tabular}

TRB3, tribbles homolog 3; CHOP, CCAAT/enhancer binding protein homologous protein; shRNA, short hairpin RNA.

Biotech Co., Ltd.) and horseradish peroxidase-conjugated goat anti-rabbit secondary antibody (1:2,000; cat no. SC-2004; Santa Cruz Biotechnology Inc., Dallas, TX, USA). Following washing in TBST, immunobands were visualized using an enhanced chemiluminescence kit (GE Healthcare, Chicago, IL, USA) following exposure to X-ray films. The western blot bands were evaluated using Fusion software (version 8.0; Vilber Lourmat, Marne-la-Vallée, France) to determine the protein expression levels and analyzed using the Quantity One program (version 4.6.9; Bio-Rad Laboratories, Inc., Hercules, CA, USA). All experiments were repeated three times.

Statistical analysis. Data are presented as the mean \pm standard deviation. All statistical analyses were performed using SPSS (version 19.0; IBM Corp., Armonk, NY, USA). Cell

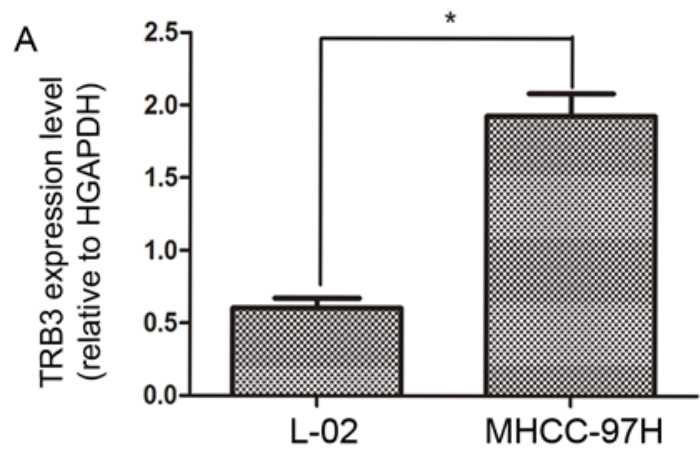

B
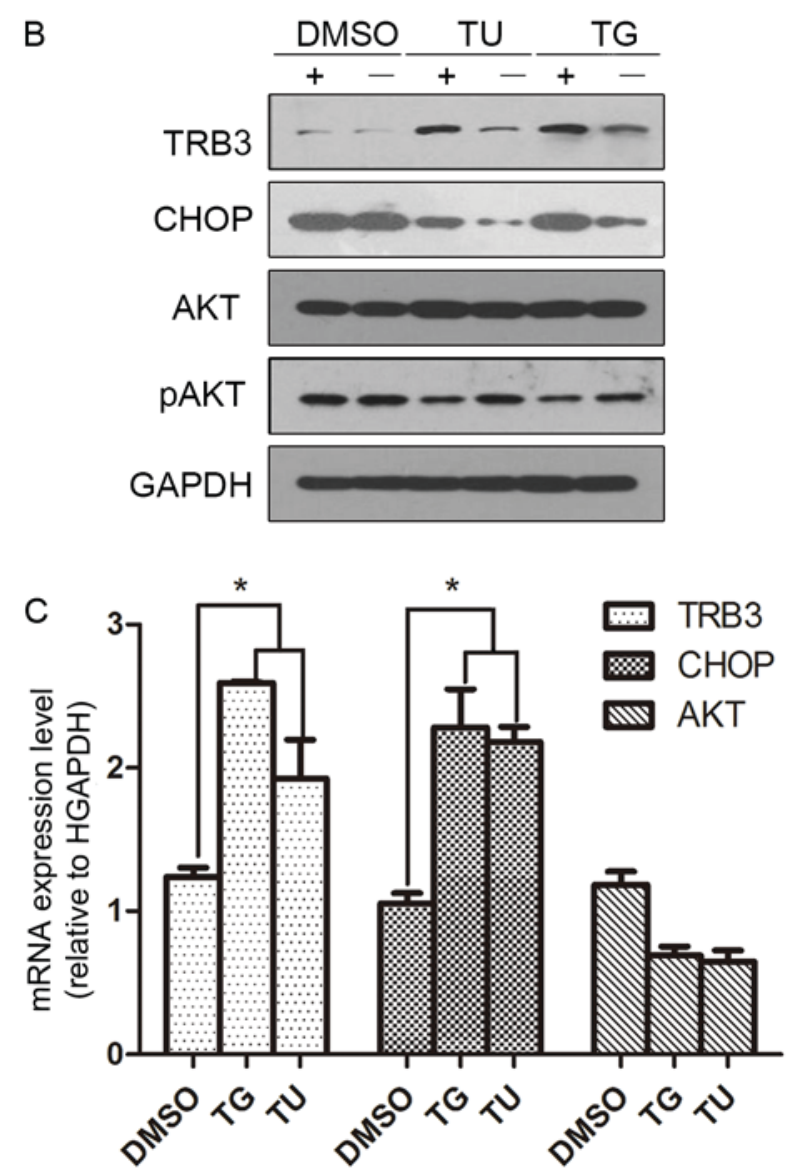

Figure 1. Levels of TRB3 mRNA expression in HCC cells and levels of TRB3, AKT and CHOP protein expression ER stress. (A) TRB3 mRNA was overexpressed in MHCC $97 \mathrm{H}$ HCC cells compared with L-02 normal hepatic cells. (B) MHCC97H cells were cultured with or without TG $(5 \mu \mathrm{mol} / 1)$ or TU $(5 \mu \mathrm{g} / \mathrm{ml})$ for $24 \mathrm{~h}$. The levels of TRB3, CHOP, AKT, pAKT and GAPDH expression were analyzed by western blotting. DMSO was used as control. (C) The levels of mRNA expression of TRB3, CHOP and AKT in MHCC $97 \mathrm{H}$ and L-02 cells were determined by reverse transcription-quantitative polymerase chain rection. DMSO was used as control. ${ }^{\text {}} \mathrm{P}<0.05$. TG, thapsigargin; TU, tunicmycin; DMSO, dimethylsulfoxide; TRB3, tribbles homolog 3; AKT, protein kinase B; CHOP, CCAAT/enhancer binding protein homologous protein; $\mathrm{p}$, phosphorylated; HCC, hepatocellular carcinoma.

proliferation analysis between the experimental and control groups was performed using unpaired t-test. The results from RT-qPCR and western blot analysis were analyzed using one-way analysis of variance. Multiple comparisons between the groups were performed using the Student-Newman-Keuls method. $\mathrm{P}<0.05$ was considered to indicate a statistically significant difference. 
A

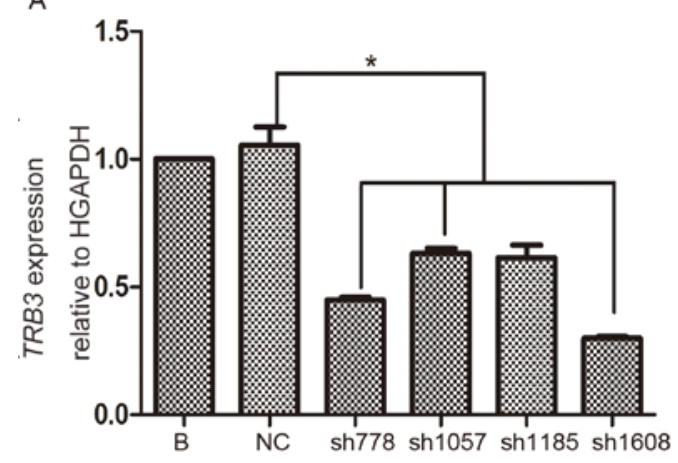

C
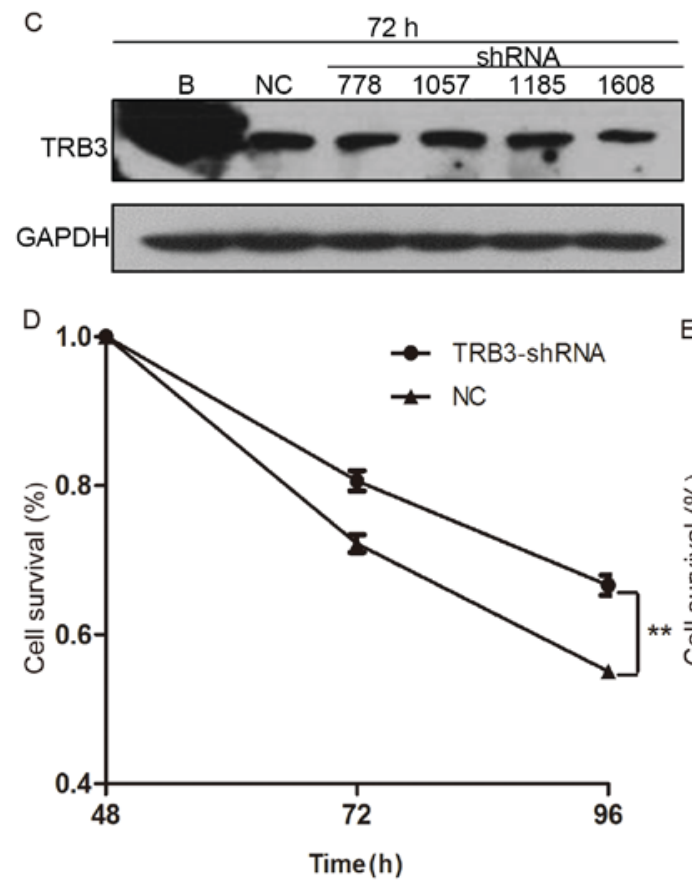
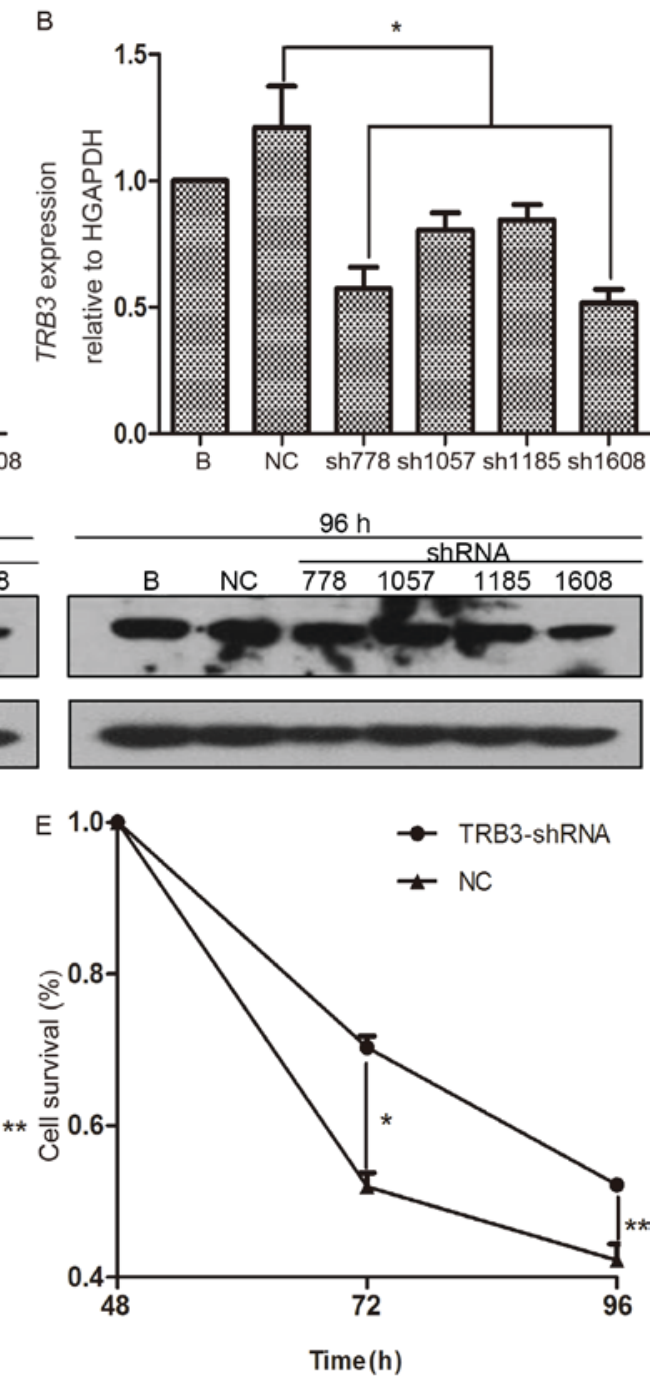

Figure 2. Knockdown of TRB3 decreases apoptosis of MHCC97H cells and reverses chemotherapy resistance. (A) Following cultivation with DMEM supplemented with 10\% FBS for 72 and $96 \mathrm{~h}$, the levels of TRB3 mRNA expression in MHCC97H cells transfected with lentivirus containing TRB3-sh778, TRB3-sh1057, TRB3-sh1185, TRB3-sh1608 or NC-shRNA for $24 \mathrm{~h}$ were detected as indicated by histogram. (B) Following cultivation with DMEM supplemented with 10\% FBS for 72 and $96 \mathrm{~h}$, the level of TRB3 mRNA expression in MHCC97H cells transfected with lentivirus containing TRB3-sh778, TRB3-sh1057, TRB3-sh1185, TRB3-sh1608 or NC-shRNA for $24 \mathrm{~h}$ was detected as presented by histogram. (C) The levels of TRB3 protein expression in MHCC97H cells were evaluated by western blot analysis. (D) Survival of MHCC97H cells transducted with the most efficient lentivirus containing TRB3- and NC-shRNA for $24 \mathrm{~h}$ were analyzed at 48,72 and $96 \mathrm{~h}$ by CCK- 8 assay. (E) TRB3- and NC-shRNA MHCC $97 \mathrm{H}$ cells were treated with cisplatin at $4 \mu \mathrm{g} / \mathrm{ml}$ for 24 h. CCK-8 assay was used to determine cell survival at 48,72 and 96 h. ${ }^{*} \mathrm{P}<0.05,{ }^{* *} \mathrm{P}<0.01$. TRB3, tribbles homolog 3; CCK-8, Cell Counting kit-8; HCC, hepatocellular carcinoma; DMEM, Dulbecco's modified Eagle's medium; FBS, fetal bovine serum; sh, short hairpin; NC, negative control; B, blank control.

\section{Results}

TRB3 was overexpressed in MHCC97H HCC cells. The level of TRB3 mRNA expression was previously demonstrated to be altered in various tumor tissues including liver cancer tissue samples from patients and HCC cell lines (HepG2, Hep3B, Huh-7, HLE and HCF) compared with normal cell lines (12). The present study demonstrated an elevated expression level of TRB3 in MHCC97H cells, 3.8 -fold compared with the expression in the L-02 normal hepatic cell line (Fig. 1A). It was revealed that TRB 3 mRNA expression level was also altered in the MHCC97H HCC cell line compared to L-02 cells.

Expression of TRB3, AKT and CHOP is induced by ER stress. To detect the changes in the expression of TRB3, CHOP and AKT under ER stress, MHCC97H cells were treated with two drugs, TG $(5 \mu \mathrm{mol} / \mathrm{l})$ and TU $(5 \mu \mathrm{g} / \mathrm{ml})$, which are known inducers of ER stress. After $24 \mathrm{~h}$, western blotting was performed to analyze the levels of TRB3, CHOP, $\mathrm{AKT}$ and pAKT expression. Following treatment with TU or TG, the expression of TRB3 and CHOP was upregulated, whereas pAKT expression was decreased compared to the DMSO control (Fig. 1B). The levels of mRNA expression of TRB3, CHOP and AKT in these cells were also determined by RT-qPCR. The results revealed that the expression levels of CHOP and TRB3 mRNA were increased following treatment with TG or TU. By contrast, the changes in the levels of AKT expression following treatment with TG or TU were not statistically significant (Fig. 1C).

Knockdown of TRB3 decreases apoptosis of HCC cells and reverses resistance to chemotherapy. To investigate the function 



Figure 3. Silencing of CHOP decreased the expression level of TRB3. (A) Following cultivation with DMEM supplemented with $10 \%$ FBS for 72 and 96 h, the level of TRB3 mRNA expression in MHCC97H cells transfected with lentivirus containing CHOP-sh342, CHOP-sh807, CHOP-sh814, CHOP-sh 929 or NC-shRNA for $24 \mathrm{~h}$ as indicated by histogram. (B) Following cultivation with DMEM supplemented with 10\% FBS for 72 and $96 \mathrm{~h}$, the level of TRB3 mRNA expression in MHCC97H cells transducted with lentivirus containing CHOP-sh342, CHOP-sh807, CHOP-sh814, CHOP-sh 929 or NC-shRNA for $24 \mathrm{~h}$ was detected. The results are presented in a histogram. (C) The levels of CHOP protein expression in MHCC97H cells were investigated by western blot analysis. (D) The level of TRB3 mRNA expression in MHCC97H cells transfected with the most effcient lentivirus containing CHOP-shRNA and NC-shRNA for $24 \mathrm{~h}$ as presented by histogram. (E) MHCC97H cells were analyzed by western blotting. ${ }^{*} \mathrm{P}<0.05,{ }^{* *} \mathrm{P}<0.01$. AKT, protein kinase $\mathrm{B}$; TRB3, tribbles homolog 3 ; CHOP, CCAAT/enhancer binding protein homologous protein; DMEM, Dulbecco's modified Eagle's medium; FBS, fetal bovine serum; sh, short hairpin; NC, negative control; B, Blank control.

of TRB3 in apoptosis of MHCC97H cells and resistance to chemotherapy, TRB3 was knocked down in the present study by transduction of MHCC97H cells with specific shRNAs. A total of four shRNA sequences targeting various regions of TRB3 mRNA (shRNA-778, shRNA-1507, shRNA-1185 and shRNA-1608) and a single NC shRNA sequence were used, and the knockdown efficiency was subsequently determined. Following lentivirus-mediated transduction for $24 \mathrm{~h}$, the levels of TRB3 expression after 72 and $96 \mathrm{~h}$ were determined in the present study. Compared with the NC control-transduced cells, transduction with shRNA-778 or shRNA-1608 was able to inhibit TRB3 expression at 72 and $96 \mathrm{~h}$ (Fig. 2A and B). In the present study, the levels of TRB3 protein expression in the transducted cells were also analyzed by western blotting and it was indicated that the level of TRB3 protein expression in shRNA-1608-trasnfected cells was markedly downregulated compared with the expression in the the $\mathrm{NC}$ control-transduced cells. (Fig. 2C). Based on these results, the most efficient shRNA sequences for inhibiting TRB3 expression were selected for further experiments.

Following shRNA transfection, CCK-8 assay was used to determine cell survival. The 24 to $96 \mathrm{~h}$ cell survival curve revealed that the number of living cells was markedly reduced when TRB3 expression was knocked down compared with the NC-shRNA-transduced cells (Fig. 2D). In addition, to investigate whether the silencing of TRB3 altered the sensitivity of MHCC97H cells to chemotherapeutic agents, TRB3- and NC-shRNA-transduced cells were treated with $4 \mu \mathrm{g} / \mathrm{ml}$ cisplatin for $24 \mathrm{~h}$ and cell survival was determined by CCK- 8 assay. There was a significantly higher number of surviving cells in the cells transduced with TRB3-shRNA compared with cells transduced with NC-shRNA following treatment with anticancer drug cisplatin for $96 \mathrm{~h}$ (Fig. 2E). These results suggested that expression of TRB3 may increase apoptosis of MHCC97H cells and reverse chemotherapy resistance.

Silencing of CHOP decreases the level of TRB3 expression. The aforementioned ER stress activation experiment confirmed the previous hypothesis that TRB3 expression was upregulated under ER stress conditions and is associated with activation of AKT. For the next part of the present study, CHOP, a well-known molecule involved in ER stress, was knocked down using the same methods for TRB3 knockdown (Figs. 3A-C). Following transduction of CHOP-shRNA, the levels of TRB3 mRNA and 

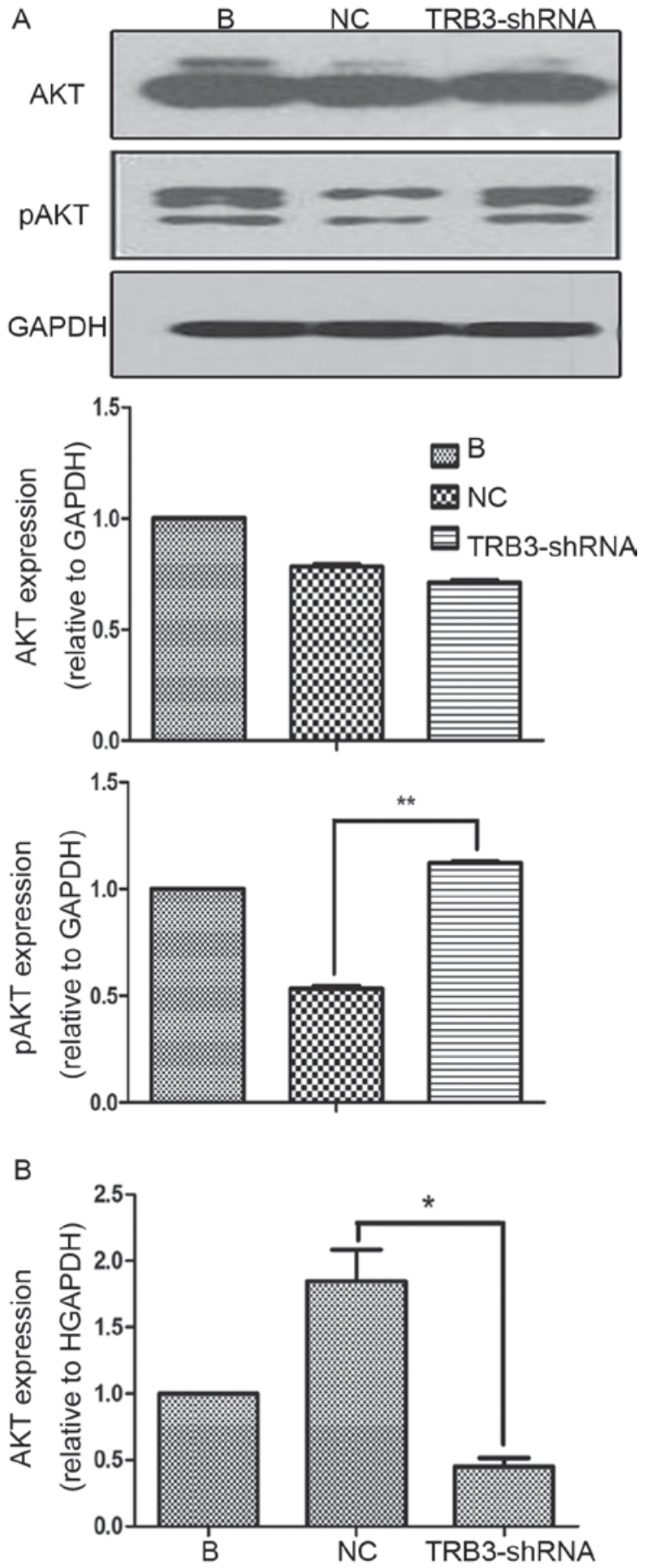

Figure 4. Loss of TRB3 increased the phosphorylation of AKT in HCC cells. (A) AKT and pAKT expression levels in cells transfected with the most efficient lentivirus containing TRB3- and NC-shRNA for $24 \mathrm{~h}$ were analyzed by western blot anlysis. (B) Analysis of TRB3 mRNA expression levels in MHCC97H cells transfected with lentivirus containing TRB3- and NC-shRNA by reverse transcription-quatitative polymerase chain reaction. ${ }^{*} \mathrm{P}<0.05,{ }^{* *} \mathrm{P}<0.01$. AKT, protein kinase B; TRB 3, tribbles homolog 3; p, phosphorylated; HCC, hepatocellular carcinoma; $\mathrm{NC}$, negative control; sh, short hairpin; B, blank control.

protein expression in MHCC97H cells were determined by RT-qPCR and western blotting, respectively. The results indicated that TRB3 expression was significantly downregulated compared with the control group (Fig. 3D and E). These results do not support the hypothesis that ER stress marker CHOP increases the expression level of TRB3.
Loss of TRB3 increases the phosphorylation of AKT in HCC cells. In the present study, it was also investigated whether increased tumorigenic properties as a result of inhibition of TRB3 expression, relied on increased AKT activation. Therefore, TRB3 expression was knocked down in $\mathrm{MHCC} 97 \mathrm{H}$ cells and activation of AKT was investigated. It was indicated that knockdown of TRB3 did not significantly affect the level of AKT expression but significantly increased the level of pAKT compared with the expression in NC shRNA-transducted cells, which suggested that TRB3 was able to prevent AKT activation (Fig. 4A). However, RT-qPCR results revealed that AKT mRNA decreased in TRB3-knocked down MHCC97H cells (Fig. 4B). These results suggested that TRB3 exerted its inhibitory effects on AKT activity at a post-transcriptional level.

\section{Discussion}

TRB3 has been recognized as an oncogene for various types of tumor, including breast cancer, colorectal cancer, giloma, oral tongue squamous cell carcinoma and HCC (12-16). A previous study revealed that TRB3 was highly expressed in a number of HCC cell lines under normal conditions or ER stress (12). To the best of our knowledge, the present study identified the first time that the target gene TRB3 is overexpressed in $\mathrm{MHCC} 97 \mathrm{H}$ HCC cells in normal conditions. Furthermore, the levels of TRB3 protein and mRNA expression were upregulated under ER stress.

Chemotherapeutic agents, including cisplatin, are usually offered to unresected patients. However, the response rates are low with rates of $\sim 20 \%$ or lower (17). In the present study, it was revealed that the downregulation of TRB3 resulted in increased survival of HCC cells and that knockdown of TRB3 was able to reverse resistance to chemotherapy in HCC cells, which suggests that poor chemotherapy effects may be associated with TRB3 expression. To summarize, these results implied that TRB3 may serve a key role in HCC.

Of note, the results from the present study indicated that TRB3 was a mediator between ER stress and AKT signaling pathways. ER stress is a protective response when the microenvironment outside the cell has a low $\mathrm{pH}$, oxygen or glucose level. When these changes are more marked, the unfolded protein response (UPR) then becomes a self-destructive signal for cell death (18). Among the three UPR downstream molecules, it is the PRKR-like endoplasmic reticulum kinase, which is upstream of AFT4-CHOP, that serves an important role in survival and apoptosis of HCC cells (19). A previous study in 2005 investigated the mechanism underlying TRB3 and apoptosis of liver cells (20). The study indicated that TRB3 is a novel target of CHOP and downregulates its own induction by the repression of CHOP/ATF4 functions, and is involved in CHOP-dependent cell death during ER stress (20). In addition, a previous study revealed that TRB3 disrupts insulin signal by binding directly to Akt and blocking PKB/AKT activation (8). Recently, it was demonstrated that salinomycin stimulated endoplasmic reticulum stress and mediated autophagy via the ATF4-CHOP-TRIB3-AKT1-mammalian target of rapamycin cascades in human non-small-cell lung cancer cells (21).

In the present study, it was revealed that CHOP and TRB3 were co-overexpressed when under ER stress and stimulated 
by thapsigargin and tunicamycin. Furthermore, the downregulation of CHOP by shRNA inhibited the expression of TRB3. Finally, the knockdown of TRB3 was able to increase the phosphorylation of AKT. Conversely, the suppression of TRB3 was able to increase AKT phosphorylation in $\mathrm{MHCC} 97 \mathrm{H}$ cells, which suggested that TRB3 may inhibit AKT activation. Of note, the present study demonstrated that AKT mRNA was decreased in TRB3-knockdown MHCC97H cells. This was in contrast to the findings of a previous study, which suggested that the level of ATK mRNA expression may be upregulated under TRB3 suppression (10). This may be attributed to the specificity of the tumor type. Consequently, the results of the present study revealed a potential underlying mechanism between ER stress-dependent cell death and the AKT signaling pathway of HCC cells.

In conclusion, the present study suggested a crucial function of TRB3, which serves a key role in regulating apoptosis and chemotherapy resistance in HCC cells. TRB3 may be a mediator for regulating ER stress-induced tumor behaviors via modulation of the AKT signal in HCC cells. The present study provided evidence that TRB3 serves an important role in increasing tumorigenesis and was a potential therapeutic target for the development of therapies for HCC.

\section{Acknowledgements}

The present study was funded by the National Natural Science Foundation of China (grant no. 81072007).

\section{References}

1. Torre LA, Bray F, Siegel RL, Ferlay J, Lortet-Tieulent J and Jemal A: Global cancer statistics, 2012. CA Cancer J Clin 65: 87-108, 2015

2. Chen W, Zheng R, Baade PD, Zhang S, Zeng H, Bray F, Jemal A, Yu XQ and He J: Cancer statistics in China, 2015. CA Cancer J Clin, 2016 doi: $10.3322 /$ caac. 21338.

3. Mizukoshi E, Nakagawa H, Kitahara M, Yamashita T, Arai K, Sunagozaka H, Iida N, Fushimi K and Kaneko S: Phase I trial of multidrug resistance-associated protein 3-derived peptide in patients with hepatocellular carcinoma. Cancer Lett 369: 242-249, 2015.

4. Mayumi-Matsuda K, Kojima S, Suzuki H and Sakata T: Identification of a novel kinase-like gene induced during neuronal cell death. Biochem Biophys Res Commun 258: 260-264, 1999.

5. Meng X, Leyva ML, Jenny M, Gross I, Benosman S, Fricker B, Harlepp S, Hébraud P, Boos A, Wlosik P, et al: A ruthenium-containing organometallic compound reduces tumor growth through induction of the endoplasmic reticulum stress gene CHOP. Cancer Res 69: 5458-5466, 2009.

6. Xu J, Lv S, Qin Y, Shu F, Xu Y, Chen J, Xu BE, Sun X and Wu J: TRB3 interacts with CtIP and is overexpressed in certain cancers. Biochim Biophys Acta 1770: 273-278, 2007.

7. Bromati CR, Lellis-Santos C, Yamanaka TS, Nogueira TC Leonelli M, Caperuto LC, Gorjão R, Leite AR, Anhê GF and Bordin S: UPR induces transient burst of apoptosis in islets of early lactating rats through reduced AKT phosphorylation via ATF4/CHOP stimulation of TRB3 expression. Am J Physiol Regul Integr Comp Physiol 300: R92-R100, 2011.
8. Du K, Herzig S, Kulkarni RN and Montminy M: TRB3: A tribbles homolog that inhibits Akt/PKB activation by insulin in liver. Science 300: 1574-1577, 2003.

9. Meric-Bernstam F, Akcakanat A, Chen H, Do KA, Sangai T, Adkins F, Gonzalez-Angulo AM, Rashid A, Crosby K, Dong M, et al: PIK3CA/PTEN mutations and Akt activation as markers of sensitivity to allosteric mTOR inhibitors. Clin Cancer Res 18: 1777-1789, 2012

10. Samarin J, Laketa V, Malz M, Roessler S, Stein I, Horwitz E, Singer S, Dimou E, Cigliano A, Bissinger $\mathrm{M}$, et al: $\mathrm{PI} 3 \mathrm{~K} / \mathrm{AKT} / \mathrm{mTOR}$-dependent stabilization of oncogenic far-upstream element binding proteins in hepatocellular carcinoma cells. Hepatology 63: 813-826, 2016.

11. Livak KJ and Schmittgen TD: Analysis of relative gene expression data using real-time quantitative PCR and the 2(-Delta Delta C(T)) method. Methods 25: 402-408, 2001.

12. Miyoshi N, Ishii H, Mimori K, Takatsuno Y, Kim H, Hirose H, Sekimoto M, Doki Y and Mori M: Abnormal expression of TRIB3 in colorectal cancer: A novel marker for prognosis. Br J Cancer 101: 1664-1670, 2009.

13. Wennemers M, Bussink J, Scheijen B, Nagtegaal ID, van Laarhoven HW, Raleigh JA, Varia MA, Heuvel JJ, Rouschop KM, Sweep FC and Span PN: Tribbles homolog 3 denotes a poor prognosis in breast cancer and is involved in hypoxia response. Breast Cancer Res 13: R82, 2011.

14. Carracedo A, Lorente M, Egia A, Blázquez C, García S, Giroux V, Malicet C, Villuendas R, Gironella M, González-Feria L, et al: The stress-regulated protein $\mathrm{p} 8$ mediates cannabinoid-induced apoptosis of tumor cells. Cancer Cell 9: 301-312, 2006.

15. Zhang J, Wen HJ, Guo ZM, Zeng MS, Li MZ, Jiang YE, He XG and Sun CZ: TRB3 overexpression due to endoplasmic reticulum stress inhibits AKT kinase activation of tongue squamous cell carcinoma. Oral Oncol 47: 934-939, 2011.

16. Ord T, Ord D, Kõivomägi M, Juhkam K and Ord T: Human TRB3 is upregulated in stressed cells by the induction of translationally efficient mRNA containing a truncated 5'-UTR. Gene 444: 24-32, 2009.

17. Yeo W, Mok TS, Zee B, Leung TW, Lai PB, Lau WY, Koh J, Mo FK, Yu SC, Chan AT, et al: A randomized phase III study of doxorubicin versus cisplatin/interferon alpha-2b/doxorubicin/fluorouracil (PIAF) combination chemotherapy for unresectable hepatocellular carcinoma. J Natl Cancer Inst 97: 1532-1538, 2005.

18. Wang WA, Groenendyk J and Michalak M: Endoplasmic reticulum stress associated responses in cancer. Biochim Biophys Acta 1843: 2143-2149, 2014

19. Mondal D, Mathur A and Chandra PK: Tripping on TRIB3 at the junction of health, metabolic dysfunction and cancer. Biochimie 124: 34-52, 2016.

20. Ohoka N, Yoshii S, Hattori T, Onozaki K and Hayashi H: TRB3, a novel ER stress-inducible gene, is induced via ATF4-CHOP pathway and is involved in cell death. EMBO J 24: 1243-1255, 2005.

21. Dim DC, Jiang F, Qiu Q, Li T, Darwin P, Rodgers WH and Peng HQ: The usefulness of S100P, mesothelin, fascin, prostate stem cell antigen and 14-3-3 sigma in diagnosing pancreatic adenocarcinoma in cytological specimens obtained by endoscopic ultrasound guided fine-needle aspiration. Diagn Cytopathol 42: 193-199, 2014. 